\title{
A basis for the non-crossing partition lattice top homology
}

\author{
Eliana Zoque
}

Received: July 31, 2003 / Revised: September 14, 2005 / Accepted: September 15, 2005

(C) Springer Science + Business Media, Inc. 2006

\begin{abstract}
We find a basis for the top homology of the non-crossing partition lattice $T_{n}$. Though $T_{n}$ is not a geometric lattice, we are able to adapt techniques of Björner (A. Björner, On the homology of geometric lattices. Algebra Universalis 14 (1982), no. 1, 107-128) to find a basis with $C_{n-1}$ elements that are in bijection with binary trees. Then we analyze the action of the dihedral group on this basis.
\end{abstract}

Keywords Non-crossing partition. Binary trees. Homology group. Catalan numbers. Representation matrix $\cdot$ Dihedral group. Stack-sortable permutations

\section{Preliminaries}

Let $\Pi_{n}$ be the set of all partitions of the set $[n]=\{1,2, \ldots, n\}$. Elements $Y$ of $\Pi_{n}$ are denoted by $Y=B_{1} / \cdots / B_{k}$, where the subsets $B_{1}, \ldots, B_{k}$ partition $[n]$ and are called the blocks of $Y$. With the refinement ordering, $X \leq Y$ if each block of $X$ is contained in a block of $Y$, and the rank function, $r_{\Pi_{n}}\left(B_{1} / \cdots / B_{k}\right)=n-k$, the set $\Pi_{n}$ is a ranked lattice. Two disjoint subsets $A$ and $B$ of $[n]$ are said to be crossing if there are $a, b \in A$ and $x, y \in B$ such that $a<x<b<y$ or $x<a<y<b$. A partition $Y=B_{1} / \cdots / B_{k}$ of $[n]$ is called non-crossing if no two of its blocks cross. Let $T_{n}$ denote the ranked lattice of all non-crossing partitions of $[n]$ ordered by refinement and with the same rank function as for $\Pi_{n}$.

Let $n \geq 3$. Kreweras shows in [8] that $\mu\left(T_{n}\right)=\mu_{T_{n}}(\mathbf{0}, \mathbf{1})=(-1)^{n-1} C_{n-1}$ where $\mathbf{1}=/ 12 \cdots n /, \mathbf{0}=1 / 2 / \cdots / n$ and $C_{k}$ is the $k$-th Catalan number:

$$
C_{k}=\frac{1}{k+1}\left(\begin{array}{c}
2 k \\
k
\end{array}\right) \text {. }
$$

\footnotetext{
E. Zoque $(\bowtie)$

Departamento de Matemáticas, Universidad de los Andes, Carrera 1\#18A-10, Bogotá, Colombia, South America e-mail: elizoque@math.uchicago.edu
} 
The lattice $T_{n}$ is EL-shellable [3] and thus Cohen-Macaulay. Then $\tilde{H}_{n-3}\left(T_{n}\right)$ is a free abelian group of rank $\left|\mu\left(T_{n}\right)\right|=C_{n-1}$.

\section{NC-bases}

An element $M$ of the partition lattice $\Pi_{n}$ is an atom if $M$ has $n-1$ blocks. We write $M=/ i j$ / to denote the atom with the block $\{i, j\}$ and all other blocks singletons.

Definition 2.1. A set $B$ of atoms of $\Pi_{n}$ is an NC-base if $|B|=n-1$, the blocks of its elements do not cross pairwise and the equality $\bigvee B=\mathbf{1}$ holds in $\Pi_{n}$.

Theorem 2.2. If $B=\left\{b_{1}, \ldots, b_{n-1}\right\}$ is an $N C$-base then the equality $r_{T_{n}}\left(b_{1} \vee b_{2} \vee \cdots \vee\right.$ $\left.b_{i}\right)=i$ holds.

Proof: Since $\Pi_{n}$ is semimodular, $r_{\Pi_{n}}\left(b_{1} \vee b_{2} \vee \cdots \vee b_{i} \vee b_{i+1}\right) \leq r_{\Pi_{n}}\left(b_{1} \vee b_{2} \vee \cdots \vee\right.$ $\left.b_{i}\right)+1$. Since $B$ is an NC-base, $r_{\Pi_{n}}\left(b_{1} \vee \cdots \vee b_{n-1}\right)=r_{\Pi_{n}}(\mathbf{1})=n-1$. Therefore the numbers $r_{\Pi_{n}}\left(b_{1}\right), r_{\Pi_{n}}\left(b_{1} \vee b_{2}\right), \ldots, r_{\Pi_{n}}\left(b_{1} \vee \cdots \vee b_{n-1}\right)$ are increasing by at most 1 , starting with 1 , and ending with $n-1$. This can only happen if $r_{\Pi_{n}}\left(b_{1} \vee \cdots \vee b_{i}\right)=i$. It remains to prove that in $\Pi_{n}, b_{1} \vee \cdots \vee b_{i}$ is a non-crossing partition.

We will show by induction on $i$ that $b_{1} \vee \cdots \vee b_{i}$ is non-crossing. For $i=1$ it is clear. Suppose $x<y<z<w$ with $x, z \in B_{1}$ and $y, w \in B_{2}$ where $B_{1}$ and $B_{2}$ are blocks of $b_{1} \vee \cdots \vee b_{i}$. By hypothesis, $b_{1} \vee \cdots \vee b_{i-1}$ is non-crossing and so $B_{1}$ and $B_{2}$ cannot both be blocks of $b_{1} \vee \cdots \vee b_{i-1}$. Assume that $B_{1}$ is the union of two blocks $C_{1}, C_{2}$ of $b_{1} \vee \cdots \vee b_{i-1}$ with $x \in C_{1}$ and $z \in C_{2}$. There exists an atom $b_{i}=/ x^{\prime} z^{\prime} /$ with $x^{\prime} \in C_{1}, z^{\prime} \in C_{2}$. Because the blocks in $b_{1} \vee \cdots \vee b_{i-1}$ do not cross and $y<z<w$, it follows that $y<z^{\prime}<w$ and so we can assume that $z=z^{\prime}$. Similarly, we may assume that $x=x^{\prime}$.

Write $u \sim v$ if $/ u v /=b_{j}$ for some $j=1, \ldots, i$. There is a sequence of vertices $t_{1}, \ldots, t_{k}$ such that $y=t_{0} \sim t_{1} \sim \ldots \sim t_{k} \sim t_{k+1}=w$. Since the atoms $b_{1}, \ldots, b_{i}$ do not cross, $x<t_{0}<z$ implies $x<t_{1}<z$ and so on, so we conclude that $x<t_{k+1}<z$, a contradiction.

Definition 2.3. Let $B=\left\{b_{1}, \ldots, b_{n-1}\right\}$ be an NC-base. If $\pi \in S_{n-1}$ let $\sigma_{\pi}(B)$ in $T_{n}$ be the maximal chain given by

$$
\sigma_{\pi}(B)=\left[b_{\pi(1)}, b_{\pi(1)} \vee b_{\pi(2)}, \ldots, b_{\pi(1)} \vee b_{\pi(2)} \vee \cdots \vee b_{\pi(n-2)}\right]
$$

By Theorem 2.2 this is in fact a maximal chain in $T_{n} \backslash\{\mathbf{0}, \mathbf{1}\}$ and therefore a simplex of dimension $n-3$ in $K\left(T_{n}\right)$.

Define

$$
\rho_{B}=\sum_{\pi \in S_{n-1}}(-1)^{\pi} \sigma_{\pi}(B)
$$

where $(-1)^{\pi}$ is the sign of the permutation $\pi$.

A simple calculation proves the following

Theorem 2.4. If $B$ is an NC-base then $\partial_{n-2}\left(\rho_{B}\right)=0$ and thus $\rho_{B} \in \tilde{H}_{n-3}$. 


\section{The construction of the basis}

Recall from [12] that there is a bilinear form $\langle$,$\rangle defined on chains by$

$$
\left\langle c_{1}, c_{2}\right\rangle= \begin{cases}1 & \text { if } c_{1}=c_{2} \\ 0 & \text { otherwise }\end{cases}
$$

and extended by linearity. Using this bilinear form, the homology and comology are dual spaces. The following lemma is a useful tool for finding bases of homology and cohomology.

Lemma 3.1. Let $P$ be a finite poset. If $H_{r}(P)$ has dimension $m$ and there are elements $\rho_{1}, \rho_{2}, \ldots, \rho_{m} \in H_{r}(P)$ and $\gamma_{1}, \gamma_{2}, \ldots, \gamma_{m} \in H^{r}(P)$ such that $\left\langle\rho_{i}, \gamma_{j}\right\rangle=\delta_{i, j}$ for all $i, j=$ $1, \ldots, m$, then $\left\{\rho_{1}, \rho_{2}, \ldots, \rho_{m}\right\}$ is a basis for $H_{r}(P)$ with dual basis $\left\{\gamma_{1}, \gamma_{2}, \ldots, \gamma_{m}\right\}$ for $H^{r}(P)$.

We will use binary trees to construct a set of $C_{n-1}$ elements of $H_{n-3}\left(T_{n}\right)$ and a set of $C_{n-1}$ elements of $H^{n-3}\left(T_{n}\right)$ such that the previous lemma holds.

Definition 3.2. A binary tree is an ordered rooted tree where each node has two subtrees, which can be empty. We distinguish between the left and the right subtree. The root of the left(right) subtree is the left(right) son of the root. The vertices of a binary tree are ordered recursively, with the left subtree ordered first, then the root, and finally the right subtree.

Definition 3.3. A binary tree is a right tree if its left subtree is empty. Let $M_{n}$ be the set of all right trees.

It is well-known that $C_{n}$ is the number of binary trees with $n$ vertices. Since the root of an ordered right tree has the label 1 , there is an obvious bijection between the set $M_{n}$ of right trees with $n$ vertices and the set of binary trees with $n-1$ vertices. Thus $\left|M_{n}\right|=C_{n-1}$.

Example 3.4. The five right trees with 4 vertices are shown in Figure 1.
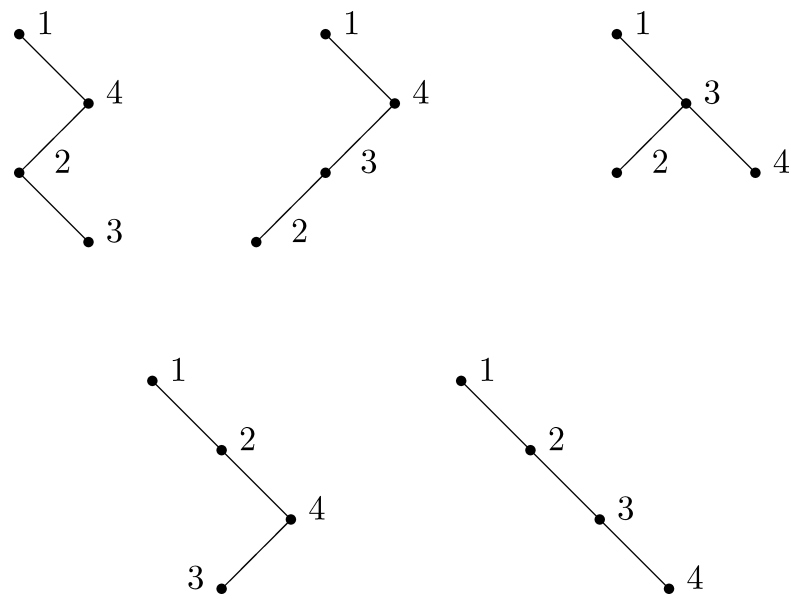

Fig. 1 All right trees with five vertices 
Enumerate the vertices of a right tree as above. Each vertex will be identified with its label. For each $A \in M_{n}$ let $B_{A}$ be the set of atoms such that $/ i j / \in B_{A}$ if and only if $(i, j)$ is an edge of $A$. In the following we shall let $/ i j /$ represent either the atom or the edge.

Theorem 3.5. If $A \in M_{n}$ then $B_{A}$ is an NC-base.

Proof: Since $A$ has $n-1$ edges, $\left|B_{A}\right|=n-1$. Since $A$ is a tree every pair of vertices is joined by a sequence of edges. This implies that $\bigvee B_{A}=\mathbf{1}$ in $\Pi_{n}$.

We will show that the elements of $B_{A}$ do not cross. Consider the atoms $/ a, c /$ and $/ b, d /$ and assume they cross. Without loss of generality suppose that $a<b<c<d$. Then $A$ has edges $(a, c)$ and $(b, d)$. In such a binary tree the root is less than every vertex in the right subtree and greater than every vertex in the left subtree. There is an edge between $a$ and $c$ and therefore $a$ is a son of $c$ or vice versa. Suppose that $c$ is a son of $a$. Then $c$ must be the right son of $a$ and, since $b$ was enumerated after $a$ and before $c, b$ must be in the left subtree of $c$. But then every vertex connected to $b$ must also be in this subtree and hence is less than or equal to $c$. Thus there cannot be an edge between $b$ and $d$. The case when $a$ is a son of $c$ is similar. It follows that the atoms of $B_{A}$ do not cross pairwise.

For brevity, let $\rho_{A}$ denote the simplex $\rho_{B_{A}}$ associated to the NC-basis $B_{A}$. Recall that $B_{A}$ is a set of atoms.

Example 3.6. Let $A$ be the right tree in Figure 2 .

The edges are: $b_{1}=(1,2), b_{2}=(2,4), b_{3}=(3,4)$.

$$
\begin{aligned}
\rho_{A}= & \left(b_{1}, b_{1} \vee b_{2}\right)-\left(b_{1}, b_{1} \vee b_{3}\right)-\left(b_{2}, b_{2} \vee b_{1}\right)+\left(b_{2}, b_{2} \vee b_{3}\right)+\left(b_{3}, b_{3} \vee b_{1}\right) \\
& -\left(b_{3}, b_{3} \vee b_{2}\right)=(12 / 3 / 4,124 / 3)-(12 / 3 / 4,12 / 34)-(1 / 24 / 3,124 / 3) \\
& +(1 / 24 / 3,1 / 234)+(1 / 24 / 3,12 / 34)-(1 / 24 / 3,1 / 234) .
\end{aligned}
$$

Definition 3.7. Enumerate the edges of a binary tree depth recursively with the edge joining the root with the left subtree first, then the edges of the left subtree, then the edge joining the root with the right subtree, and, finally, the edges of the right subtree.

Let $b_{1}, \ldots, b_{n-1}$ be the edges of $A \in M_{n}$ in this order. The characteristic chain of $A$ is $S_{A}=\left[b_{1}, b_{1} \vee b_{2}, \ldots, b_{1} \vee \cdots \vee b_{n-2}\right]$. By Theorem 2.2 it is a maximal chain in $T_{n}$.

The following lemma is clear.

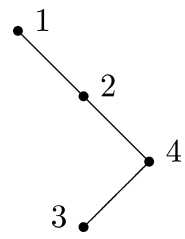

Fig. 2 A right tree

$\underline{\text { Q }}$ Springer 
Lemma 3.8. For every $j=1, \ldots, n-1$ the edges $b_{1}, \ldots, b_{j}$ form a connected component of $A$, which contains the root.

Lemma 3.9. Every block of $b_{1} \vee \cdots \vee b_{k}$ has only one element, except the one containing the vertex 1 . If $a>1$ belongs to this block, then the father of a also belongs to it.

Proof: The first part is clear from Lemma 3.8. Suppose $a>1$ belongs to the connected component but its father $b$ does not. Then there is a path from $a$ to 1 which does not contain the vertex $b$. But this implies that there is a cycle in $A$ contradicting that $A$ is a tree.

Theorem 3.10. $\left\langle S_{A}, \rho_{A^{\prime}}\right\rangle=\delta_{A, A^{\prime}}$

Proof: It is clear from the definition of $\rho_{A}$ that $S_{A}$ is a chain of $\rho_{A}$ and that it appears with the sign + . Assume that $\pm S_{A}$ is a chain of $\rho_{A^{\prime}}$ and that the atoms of $\rho_{A}$ and $\rho_{A}^{\prime}$ are $b_{1}, b_{2}, \ldots, b_{n-1}$ and $b_{1}^{\prime}, b_{2}^{\prime}, \ldots, b_{n-1}^{\prime}$, respectively (ordered by depth). Then there is a permutation $k_{1}, k_{2}, \ldots, k_{n-1}$ of $1,2, \ldots, n-1$ such that $b_{1} \vee \cdots \vee b_{m}=b_{k_{1}}^{\prime} \vee \cdots \vee b_{k_{m}}^{\prime}$ for $m=1, \ldots, n-1$.

We will show, by induction on $m$, that $b_{m}=b_{k_{m}}^{\prime}$ and that the connected component formed by the edges $b_{1}, \ldots, b_{m}$ in $A$ is equal to the connected component formed by the edges $b_{k_{1}}^{\prime}, \ldots, b_{k_{m}}^{\prime}$ in $A^{\prime}$. For $m=1$ we have $b_{1}=b_{k_{1}}^{\prime}=/ 1, i /$. Assume that $b_{1}=b_{k_{1}}^{\prime}, \ldots, b_{m-1}=$ $b_{k_{m-1}}^{\prime}$. Let $U, V$ be blocks with more than one element in $b_{1} \vee \cdots \vee b_{m-1}=b_{k_{1}}^{\prime} \vee \cdots \vee b_{k_{m-1}}^{\prime}$ and $b_{1} \vee \cdots \vee b_{m}=b_{k_{1}}^{\prime} \vee \cdots \vee b_{k_{m}}^{\prime}$, respectively (they exist by Lemma 3.9). Then $V=$ $U \cup\{x\}$, where $b_{m}=/ x, y /, b_{k_{m}}^{\prime}=/ x, z /$, with $y, z \in U$. It must happen that, in the tree $A^{\prime}, x$ is a son of $z$ or vice versa. But $z \in U$, and if $z$ is a son of $x$ then $x \in U$, which is impossible. Therefore $x$ is a son of $z$ in $A^{\prime}$. In the same way, $x$ is a son of $y$ in $A$. Let $E$ be the tree formed by the edges $b_{1}, \ldots, b_{m-1}$. The vertices of this tree are the elements of $U$. Consider the trees $F$ and $F^{\prime}$ whose edges are $b_{1}, \ldots, b_{m}$ and $b_{k_{1}}^{\prime}, \ldots, b_{k_{m}}^{\prime}$, respectively. These two trees are obtained by adding the edges $b_{m}$ and $b_{k_{m}}^{\prime}$, respectively, to the tree $E$ and therefore they can only differ in the edge containing the vertex $x$. Assume that $y \neq z$. Let $1=a_{1}, a_{2}, \ldots, a_{t}=x, 1=a_{1}^{\prime}, a_{2}^{\prime}, \ldots, a_{s}^{\prime}=x$ be the paths from 1 to $x$ in the trees $F$ and $F^{\prime}$, respectively. We have $a_{t-1}=y, a_{s-1}^{\prime}=z$. Let $j=\max \left\{l \mid a_{l}=a_{l}^{\prime}\right\}<\min \{s-1, t-1\}$. Then $a_{j}=a_{j}^{\prime}, a_{j+1} \neq a_{j+1}^{\prime}$. This situation is shown in Figure 3 .

Since $j+1<s, t, a_{j+1} \neq x$ and $a_{j+1}^{\prime} \neq x$ and therefore $a_{j+1}$ and $a_{j+1}^{\prime}$ are in the tree $E$, given that $a_{j+1}$ and $a_{j+1}^{\prime}$ are sons of $a_{j}=a_{j}^{\prime}$.

Without loss of generality we may assume that $x<a_{j}$. Then $a_{j+1}$ must be the left son of $a_{j}$ in $E$. In the same way, $a_{j+1}^{\prime}$ must be the left son of $a_{j}^{\prime}=a_{j}$ in $E$, which contradicts that $a_{j+1} \neq a_{j+1}^{\prime}$. We conclude that $y=z$ and therefore $b_{m}=b_{k_{m}}^{\prime}$. Finally, when we adjoin the same edge to two equal connected components, we obtain the same connected component. This completes the induction.

The next theorem follows from Lemma 3.1.

Theorem 3.11. $\left\{\rho_{A} \mid A \in M_{n}\right\}$ is a basis of $\tilde{H}_{n-3}\left(T_{n}\right)$.

Note that this base is different from the one that is obtained from an EL-labeling because there is no maximal chain that is shared by all the elements of the basis. In fact, every atom belongs to at least one chain.

In [12], several bases for $H\left(\Pi_{n}\right)$ are described, using node labeled trees. Although our basis is described in terms of node labeled trees and the construction itself is almost the same, 
the trees in Wachs' article are increasing (meaning that every vertex except the root is less than its father) while the binary trees used here are not. It is the fact that the trees we consider here have their vertices ordered that guarantees that the partitions are non-crossing.

\section{The action of the dihedral group}

Let $D_{n}$ be the dihedral group. The elements of $D_{n}$ have the form $\tau^{a} \gamma^{b}$, with $0 \leq a \leq n-$ $1,0 \leq b \leq 1$, where $\tau$ is the rotation given by $\tau(i)=i-1$ for $2 \leq i \leq n$ and $\tau(1)=n$; and $\gamma$ is the reflection defined by $\gamma(1)=1$ and $\gamma(i)=n-i+2$. We want to analyze the action of $D_{n}$ on the homology. To do this we analyze the action of $\tau$ and $\gamma$.

It is proved in [12] that, under the hypothesis of Lemma 3.1, the representation matrix $M(g)$ for $g$ acting on $H_{r}(P)$ with respect to the basis $\left\{\rho_{1}, \ldots, \rho_{m}\right\}$ has $i, j$ component given by $M_{i, j}(g)=\left\langle g \rho_{j}, \gamma_{i}\right\rangle$. As a consequence, we have the following lemma.

Lemma 4.1. For every element $g$ in the dihedral group $D_{n}$, the entries $M_{i, j}(g)$ of its representation matrix with respect to the basis in Theorem 3.11 are $-1,0$ or 1 .

In the following sections we will give a method for calculating the representation matrices $M(g)$ with respect to the basis in Theorem 3.11 .

\subsection{Action of the reflection}

Lemma 4.2. For every tree $A \in M_{n}$ there exists a tree $D \in M_{n}$ such that $\gamma\left(\rho_{A}\right)= \pm \rho_{D}$.

Proof: Let $A \in M_{n}$, with edges $a_{1}, \ldots, a_{n-1}$, ordered by depth. Then $\gamma\left(a_{1}\right), \ldots, \gamma\left(a_{n-1}\right)$ are the edges of a tree $D=\gamma(A) \in M_{n}$. When these edges are enumerated by depth, they may appear in a different order $\gamma\left(a_{\beta(1)}\right), \ldots, \gamma\left(a_{\beta(n-1)}\right)$. Then, for every $\pi \in S_{n-1}$,

$$
\begin{aligned}
\gamma\left(\sigma_{\pi}(A)\right) & =\left[\gamma\left(a_{\pi(1)}\right), \ldots, \gamma\left(a_{\pi(1)}\right) \vee \gamma\left(a_{\pi(2)}\right) \vee \cdots \vee \gamma\left(a_{\pi(n-2)}\right)\right] \\
& =\left[\gamma\left(a_{\pi \circ \beta^{-1} \circ \beta(1)}\right), \cdots, \gamma\left(a_{\pi \circ \beta^{-1} \circ \beta(1)}\right) \vee \ldots \vee \gamma\left(a_{\pi \circ \beta^{-1} \circ \beta(n-2)}\right)\right]=\sigma_{\pi \circ \beta^{-1}}(D),
\end{aligned}
$$

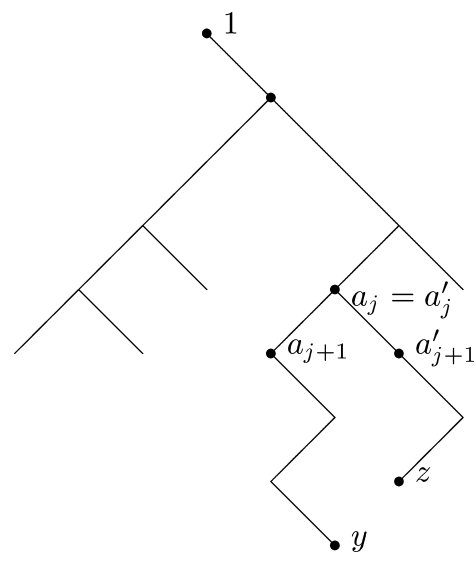

Fig. 3 An impossible situation 
and therefore

$$
\begin{aligned}
\gamma\left(\rho_{A}\right) & =\sum_{\pi \in S_{n-1}}(-1)^{\pi} \gamma\left(\sigma_{\pi}(A)\right) \\
& =\sum_{\pi \in S_{n-1}}(-1)^{\pi} \sigma_{\pi \circ \beta^{-1}}(D)=(-1)^{\beta} \sum_{\pi \in S_{n-1}}(-1)^{\pi \circ \beta^{-1}} \sigma_{\pi \circ \beta^{-1}}(D) \\
& =(-1)^{\beta} \sum_{\pi \in S_{n-1}}(-1)^{\pi} \sigma_{\pi}(D)= \pm \rho_{D} .
\end{aligned}
$$

As a consequence of this lemma, the representation matrix of $\gamma$ is the direct sum of matrices of the forms

$$
\left(\begin{array}{ll}
0 & 1 \\
1 & 0
\end{array}\right),\left(\begin{array}{cc}
0 & -1 \\
-1 & 0
\end{array}\right),(1),(-1)
$$

Example 4.3. Let $A$ be the tree on the left in Figure 4 .

The edges of $A$ are $a_{1}=(1,2), a_{2}=(2,4), a_{3}=(3,4), a_{4}=(4,5), a_{5}=(5,6)$. Then $\gamma\left(a_{1}\right)=(1,6), \gamma\left(a_{2}\right)=(4,6), \gamma\left(a_{3}\right)=(4,5), \gamma\left(a_{4}\right)=(3,4), \gamma\left(a_{5}\right)=(2,3)$. In order, the edges of $D$ are $(1,6),(4,6),(3,4),(2,3),(4,5)$ and the corresponding permutation is $\beta=12453$. Since this permutation is even, we conclude that $\gamma\left(\rho_{A}\right)=\rho_{D}$.

Now we want to find the number of times that each of the matrices given above appears in the representation matrix $M(\gamma)$. The following lemma is clear from the proof of the Lemma 4.2 .

Lemma 4.4. For $A \in M_{n}, \gamma\left(\rho_{A}\right)= \pm \rho_{A}$ if and only if the right subtree of $A$ (the tree with vertices $2,3, \ldots, n)$ is symmetric, i.e., if it is invariant under the action of $\gamma$.

Now we give a method to determine the sign in the expression $\gamma\left(\rho_{A}\right)= \pm \rho_{D}$.

In $A$, let $k$ be the son of 1 , and let $A_{l}$ and $A_{r}$ be the left and right subtrees of $k$, respectively. Then $A_{l}$ has $k-2$ vertices and $A_{r}$ has $n-k$ vertices. The edges in $A_{l}$, including the one that joins its root with $k$, are $a_{2}, \ldots, a_{k-1}$. As in the proof of the Lemma 4.2, let $\beta_{l}$ be the permutation of the edges of $A_{l}$ such that $\gamma\left(a_{\beta_{l}(2)}\right), \ldots, \gamma\left(a_{\beta_{l}(k-1)}\right)$ are the edges of $\gamma\left(A_{l}\right)$, ordered by depth. Define $\beta_{r}$ in an analogous way.
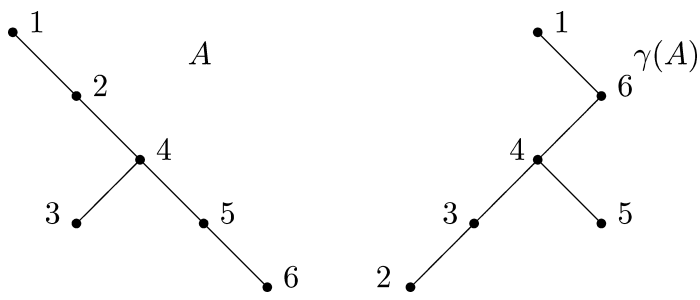

Fig. 4 A tree and its reflection 
Then the edges of $\gamma(A)$, ordered by depth, are

$$
\gamma\left(a_{\beta(1)}\right), \ldots, \gamma\left(a_{\beta(n-1)}\right)=a_{1}, \gamma\left(a_{\beta_{r}(k)}\right), \ldots, \gamma\left(a_{\beta_{r}(n)}\right), \gamma\left(a_{\beta_{l}(2)}\right), \ldots, \gamma\left(a_{\beta_{l}(k-1)}\right)
$$

From this we conclude that the sign of $\beta$, which is also the sign in the expression $\gamma\left(\rho_{A}\right)=$ $\pm \rho_{D}$, is $(-1)^{(k-2)(n-k)}(-1)^{\beta_{l}}(-1)^{\beta_{r}}$. We will use this fact in the proof of the following theorem.

Theorem 4.5. The number of trees $A \in M_{n}$ such that $\gamma\left(\rho_{A}\right)=\rho_{A}$ is $C_{(n-2) / 2}$ ifn is congruent to $2 \bmod 4$, and 0 otherwise.

The number of trees $A \in M_{n}$ such that $\gamma\left(\rho_{A}\right)=-\rho_{A}$ is $C_{(n-2) / 2}$ if $n$ is multiple of 4 , and 0 otherwise.

In other words, the multiplicity of the matrix $(-1)^{(n-2) / 2}$ in the representation matrix for $\gamma$ is $C_{(n-2) / 2}$.

Proof: If $n$ is odd, the trees in $M_{n}$ cannot be symmetric, and the result follows from Lemma 4.4. If $n$ is even, say $n=2 m$, there are $C_{m-1}$ symmetric trees (the left subtree is determined by the right one). Let $\beta, \beta_{l}$ and $\beta_{r}$ be as in the paragraph before the statement of the theorem above. Then $\beta_{l}$ and $\beta_{r}$ are inverse permutations and so they have the same sign. Thus $(-1)^{\beta}=$ $(-1)^{(m-1)^{2}}=(-1)^{m-1}$. We have concluded $\gamma\left(\rho_{A}\right)=(-1)^{(m-1)} \rho_{A}$ for every $A \in M_{n}$.

The following result will enable us to determine the multiplicities of the $2 \times 2$ matrices in $M(\gamma)$.

Theorem 4.6. Let $x_{n}$ (resp. $y_{n}$ ) be the number of $A \in M_{n}$ so that $\gamma\left(\rho_{A}\right)=+\rho_{D}$ (resp. $\left.\gamma\left(\rho_{A}\right)=-\rho_{D}\right)$ for some $D \in M_{n}$. Then

$$
x_{n}= \begin{cases}\sum_{k=2}^{n}\left(x_{k-1} x_{n-k+1}+y_{k-1} y_{n-k+1}\right), & \text { if } n=2 m+1, \\ \sum_{p=1}^{m}\left(x_{2 p-1} x_{n-2 p+1}+y_{2 p-1} y_{n-2 p+1}\right)+2 \sum_{p=1}^{m-1}\left(x_{2 p} y_{n-2 p}\right), & \text { if } n=2 m,\end{cases}
$$

and

$$
y_{n}= \begin{cases}2 \sum_{k=2}^{n} x_{k-1} y_{n-k+1}, & \text { if } n=2 m+1, \\ 2 \sum_{p=1}^{m}\left(2 x_{2 p-1} y_{n-2 p+1}\right)+\sum_{p=1}^{m-1}\left(x_{2 p} x_{n-2 p}+y_{2 p} y_{n-2 p}\right), & \text { if } n=2 m .\end{cases}
$$

Note that the multiplicity of the matrix $\left(\begin{array}{ll}0 & 1 \\ 1 & 0\end{array}\right)$ in the representation matrix of $\gamma$ is $\left(x_{n}-C_{(n-2) / 2}\right) / 2$ if $n$ congruent to $2 \bmod 4$, and $x_{n} / 2$ otherwise. A similar expresion for the multiplicity of $\left(\begin{array}{cc}0 & -1 \\ -1 & 0\end{array}\right)$ can be found.

Proof: Using the formula $(-1)^{\beta}=(-1)^{(k-2)(n-k)}(-1)^{\beta_{l}}(-1)^{\beta_{r}}$ and taking into account the parity of $n$, we consider several cases. Assume $n=2 m$ is even. Then $\gamma\left(\rho_{A}\right)=+\rho_{D}$ if $k=2 p$ 包 Springer 
is even and $\beta_{l}, \beta_{r}$ have the same sign, or if $k=2 p+1$ is odd and $\beta_{l}, \beta_{r}$ have different sign. In these cases

$$
x_{n}=\sum_{p=1}^{m}\left(x_{2 p-1} x_{n-2 p+1}+y_{2 p-1} y_{n-2 p+1}\right)+\sum_{p=1}^{m-1}\left(x_{2 p} y_{n-2 p}+y_{2 p} x_{n-2 p}\right)
$$

and

$$
y_{n}=2 \sum_{p=1}^{m}\left(x_{2 p-1} y_{n-2 p+1}\right)+\sum_{p=1}^{m-1}\left(x_{2 p} x_{n-2 p}+y_{2 p} y_{n-2 p}\right) .
$$

The other expressions are similar.

\subsection{Characteristic sequences}

In order to find the representation matrix of the rotation $\tau$ we have to introduce the tool of the characteristic sequences.

Every tree $A \in M_{n}$ with edges $\left\{b_{1}, \ldots b_{n-1}\right\}$ has a characteristic chain $S_{A}=\left[b_{1}, b_{1} \vee\right.$ $\left.b_{2}, \ldots, b_{1} \vee \cdots \vee b_{n-2}\right]$. By Lemma 3.9, for $i=1, \ldots n-2$, the block $U_{i}$ of $b_{1} \vee \cdots \vee b_{i}$ which contains the vertex 1 is the unique block with more than one element. Let $U_{0}=$ \{1\}. It is clear that $U_{i-1} \subset U_{i}$. Therefore we can order the vertices of $A$, such that $U_{i}=$ $\left\{1, a_{1}, \ldots a_{i-1}, a_{i}\right\}$. This ordering of the vertices is called a pre-order. It can be obtained recursively by first taking the root, then the vertices of the left subtree, and finally the vertices of the right subtree.

Definition 4.7. The sequence $1, a_{1}, \ldots a_{n-1}, a_{n}$ is called the characteristic sequence of the tree A.

Example 4.8. The characteristic sequence of the tree $A$ shown in Figure 4 is $1,2,4,3,5,6$.

Definition 4.9. The stack-sorting $S$ of a sequence of diferent numbers is defined recursively as follows: $S(\emptyset)=\emptyset$ and $S\left(A_{1} x A_{2}\right)=S\left(A_{1}\right) S\left(A_{2}\right) x$ if $x$ is the largest element of the sequence $A_{1} x A_{2}$. A permutation $\sigma \in S_{n}$ (considered as a sequence) is called stack-sortable if $S(\sigma)=$ $1,2, \ldots, n$.

There is a well-known correspondence between binary trees and stack-sortable permutations that can be found in [7]. As a consequence, we have the following result.

Theorem 4.10. A sequence $1, a_{1}, \ldots a_{n-1}$ is the characteristic sequence of some $A \in M_{n}$ if and only if it is stack-sortable and $\left\{a_{1}, \ldots a_{n-1}\right\}=\{2, \ldots, n\}$.

Note that the number 1 at the beginning of the sequence forces the left subtree to be empty and guarantees that the tree is a right tree.

The following lemma, which can be found in [7], will be useful later.

Lemma 4.11. If $a_{1}, \ldots a_{m}$ is a stack-sortable permutation, then there is no triple of indices $i<j<k$ so that $a_{k}<a_{i}<a_{j}$. 


\subsection{Action of the rotation}

We are going to use the result from [12] mentioned at the beginning of this section. Let $A \in M_{n}$ with edges $b_{1}, \ldots, b_{n}$, ordered by depth. Let $b_{i}^{\prime}=\tau\left(b_{i}\right)$. Note that $b_{1}^{\prime}, \ldots, b_{n}^{\prime}$ are the edges of a binary tree $A^{\prime}$ with ordered vertices. The root is $\tau(1)=n$, the right subtree is empty, and the left subtree is obtained by subtracting 1 from the vertices of the right subtree of $A$. This is because $\tau$ switches the root with every other vertex and leaves the ordering of the other vertices unchanged. An example is shown in Figure 5.

If $t_{G} \neq 0$ and the edges of $G$ (ordered by depth) are $d_{1}, \ldots, d_{n-1}$ then $S_{G}$ appears in

$$
\tau\left(\rho_{A}\right)=\sum_{G \in M_{n}} t_{G} \rho_{G}=\tau\left(\sum_{\pi \in S_{n}}(-1)^{\pi} \sigma_{\pi}(A)\right)=\sum_{\pi \in S_{n}}(-1)^{\pi} \tau\left(\sigma_{\pi}(A)\right) .
$$

Thus there exists a permutation $\pi \in S_{n}$ with $t_{G}=(-1)^{\pi}$ and

$$
\begin{aligned}
S_{G} & =\left[d_{1}, d_{1} \vee d_{2}, \ldots, d_{1} \vee \cdots \vee d_{n-1}\right] \\
& =\tau\left(\sigma_{\pi}(A)\right)=\tau\left(\left[b_{\pi(1)}, b_{\pi(1)} \vee b_{\pi(2)}, \ldots, b_{\pi(1)} \vee b_{\pi(2)} \vee \cdots \vee b_{\pi(n-1)}\right]\right) \\
& =\left[b_{\pi(1)}^{\prime}, b_{\pi(1)}^{\prime} \vee b_{\pi(2)}^{\prime}, \ldots, b_{\pi(1)}^{\prime} \vee \cdots \vee b_{\pi(n-1)}^{\prime}\right] .
\end{aligned}
$$

By Lemma 3.9, $d_{1} \vee \cdots \vee d_{j}=b_{\pi(1)}^{\prime} \vee \cdots \vee b_{\pi(j)}^{\prime}$ has only one block with more than one element, and so $b_{\pi(1)}^{\prime}, \ldots, b_{\pi(j)}^{\prime}$ form a connected component of the tree $A^{\prime}$. Let $W_{j}$ be the block with more than one element in $d_{1} \vee \cdots \vee d_{j}=b_{\pi(1)}^{\prime} \vee \cdots \vee b_{\pi(j)}^{\prime}$ and let $1, g_{1}, \ldots, g_{n-1}$ the characteristic sequence of $G$. Then $W_{j}=\left\{1, g_{1}, \ldots, g_{j}\right\}$. From this we conclude the following.

Lemma 4.12. Let $G \in M_{n}$ with characteristic sequence $1, g_{1}, \ldots, g_{n-1}$. Then $\rho_{G}$ appears in $\tau\left(\rho_{A}\right)$ with non-zero coefficient if and only if for every $j$ the vertices $1, g_{1}, \ldots, g_{j}$ form a connected component of $A^{\prime}=\tau(A)$.

Example 4.13. Let $A$ be the left tree in Figure 5. The edges of $A$ are $b_{1}=(1,5), b_{2}=$ $(5,3), b_{3}=(3,2), b_{4}=(3,4), b_{5}=(5,6)$, and the edges of $A^{\prime}$ are $b_{1}^{\prime}=(6,4), b_{2}^{\prime}=$ $(4,2), b_{3}^{\prime}=(2,1), b_{4}^{\prime}=(2,3), b_{5}^{\prime}=(4,5)$.

The characteristic sequences for possible $G$ with $t_{G} \neq 0$ are: $1,2,3,4,5,6 ; 1,2,4,3,5$, $6 ; 1,2,3,4,6,5 ; 1,2,4,3,6,5$; and the trees (that we call $G_{1}, G_{2}, G_{3}$ and $G_{4}$, respectively) for these sequences are as shown in Figure 6.

Other sequences, like $1,2,4,6,5,3$, are also obtained from $A^{\prime}$ but they are not characteristic sequences since they are not stack-sortable. Therefore $\tau\left(\rho_{A}\right)= \pm \rho_{G_{1}} \pm \rho_{G_{2}} \pm \rho_{G_{3}} \pm \rho_{G_{4}}$.
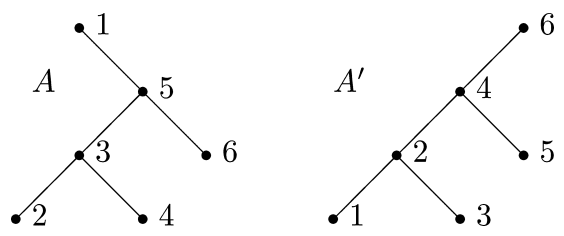

Fig. 5 A tree and its rotation 

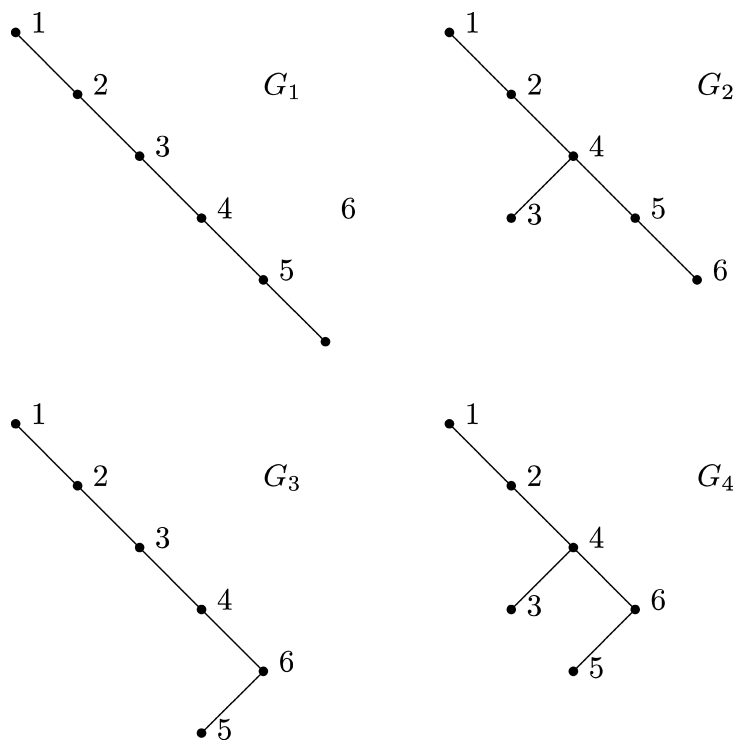

$G_{4}$

Fig. 6 The possible trees $G$

The signs can be calculated from the signs of the corresponding permutations of the edges as in the Example 4.3. Thus, in this example, $\tau\left(\rho_{A}\right)=\rho_{G_{1}}-\rho_{G_{2}}-\rho_{G_{3}}+\rho_{G_{4}}$.

The Lemma 4.15 give us a shortcut for calculations as the ones in the previous example.

Definition 4.14. Let $y_{1}=1$ and, for $i \geq 0$, let $y_{i+1}$ be the father of $y_{i}$ in $A^{\prime}$. The sequence $y_{1}, \ldots, y_{s}=n$ is called the main branch of $A^{\prime}$. Let $Y_{i}$ be the set containing the vertex $y_{i}$ and every vertex in the right subtree of $y_{i-1}$.

Since 1 has no left son in $A^{\prime}, Y_{2} \cup \cdots \cup Y_{s}=\{2, \ldots, n\}$.

Lemma 4.15. Let $\left(g_{i}\right)=1, g_{1}, \ldots, g_{n-1}$ be as in Lemma 4.12. Then the first terms of the sequence $g_{1}, \ldots, g_{n-1}$ are the elements of $Y_{2}$ in some order, followed by the elements of $Y_{3}$ in some order, and so on.

Proof: The vertex with the number $1=y_{1}$ has as father the vertex $y_{2}$, its left subtree is empty, and the vertices in its right subtree are the other elements of $Y_{2}$. Therefore the vertices adjacent to 1 belong to $Y_{2}$, so $g_{1} \in Y_{2}$. But after choosing a vertex, we must take every vertex less than it that has not been chosen before (according to Lemma 4.11), and those vertices are in $Y_{1} \cup Y_{2}$. Similarly we show that the vertices in every $Y_{i}$ appear consecutively because the vertices with label less than $y_{i}$ are in $Y_{1} \cup \cdots \cup Y_{i}$.

Example 4.16. For the tree $A$ in Example 4.13, $y_{1}=1, y_{2}=2, y_{3}=4, y_{4}=6$ and $Y_{1}=$ $\{1\}, Y_{2}=\{2\}, Y_{3}=\{4,3\}, Y_{4}=\{6,5\}$, and the possible characteristic sequences are $1,2,3$, $4,5,6 ; 1,2,3,4,6,5 ; 1,2,4,3,5,6$; and $1,2,4,3,6,5$.

In general, it is not true that every ordering of $Y_{i}$ will work: by Theorem 4.10, the sequence must be stack-sortable. 
Although this gives us an algorithm to find the representation matrix for $\tau$, it would be interesting to find a more general way to do it.

Acknowledgments The author is very grateful to Professor Carlos Montenegro for posing the question and for helpful conversations; to the referees for suggestions and references; and to Professor Arun Ram for a careful review of this paper.

\section{References}

1. A. Björner, "On the homology of geometric lattices," Algebra Universalis 14(1) (1982), 107-128.

2. A. Björner, "Orderings of Coxeter groups," Combinatorics and algebra (Boulder, Colo., 1983), 175-195, Contemp. Math., 34, Amer. Math. Soc., Providence, RI, 1984.

3. A. Björner, "Shellable and Cohen-Macaulay partially ordered sets," Trans. Amer. Math. Soc. 260, 159-183.

4. P. Edelman, "Chain enumeration and noncrossing partitions," Discrete Math. 31(2), (1980), 171-180.

5. P. Edelman, "Multichains, noncrossing partitions and trees," Discrete Math. 40(2/3), (1982), 171-179.

6. P. Edelman, R. Simion, "Chains in the lattice of noncrossing partitions," Discrete Math. 126(1/3), (1994), 107-119.

7. D. Knuth, The Art of Computer Programming, Addison-Wesley Pub. Co., Upper Saddle River, NJ, 1973.

8. G. Kreweras, "Sur les partitions non croisÈes d'un cycle," (French) Discrete Math. 1(4), (1972), 333-350.

9. C. Montenegro, The fixed point non-crossing partition lattices, Preprint, 1993.

10. V. Reiner, "Non-crossing partitions for classical reflection groups," Discrete Math. 177(1/3), (1997), $195-222$.

11. D. Rotem and Y. L. Varol, "Generation of binary trees from ballot sequences," J. Assoc. Comput. Mach. 25(3), (1978), 396-404.

12. M. Wachs, "On the (co)homology of the partition lattice and the free Lie algebra," Discrete Math. 193 (1998), 287-319. 